\title{
A phase II, randomized, single-blinded, placebo-controlled clinical trial on the efficacy of Curcumina and Calendula suppositories for the treatment of patients with chronic prostatitis/chronic pelvic pain syndrome type III
}

\author{
Giuseppe Morgia, Giorgio Ivan Russo, Daniele Urzì, Salvatore Privitera, Tommaso Castelli, \\ Vincenzo Favilla, Sebastiano Cimino \\ Urology section, Department of Surgery, University of Catania Italy.
}

\begin{abstract}
Summary Objective: The management of chronic prostatitis/chronic pelvic pain syndrome type III (CP/CPPS) has been always considered complex due to several biopsychological factors underling the disease. In this clinical study, we aimed to evaluate the efficacy of the treatment with Curcumin and Calendula extract in patients with CP/CPPS III. Material and methods: From June 2015 to January 2016 we enrolled 60 consecutive patients affected by CP/CPPS III in our institution. Patients between 20 and 50 year of age with symptoms of pelvic pain for 3 months or more before study, a total National Institutes of Health Chronic Prostatitis Symptom Index (NIH-CPSI) score $\geq 15$ point and diagnosed with NIH category III. Patients were then allocated to receive placebo (Group A) or treatment (Group B). Treatment consisted of rectal suppositories of Curcumin extract $350 \mathrm{mg}$ (95\%) and Calendula extract 80 mg (1 suppository/die for 1 month). Patients of Group B received 1 suppository/die for 1 month of placebo. The primary endpoint of the study was the reduction of NIH-CPSI. The secondary outcomes were the change of peak flow, IIEF-5, VAS score and of premature ejaculation diagnostic tool (PEDT).

Results: A total of 48 patients concluded the study protocol. The median age of the all cohort was 32.0 years, the median NIH-CPSI was 20.5, the median IIEF-5 was 18.5 , the median PEDT was 11.0, the median VAS score was 7.5 and the median peak flow was 14.0. After 3 months of therapy in group A we observed a significant improvement of NIH-CPSI (-5.5; $p<0.01)$, IIEF-5 (+ 3.5; $p<0.01)$, PEDT (-6.5; $p<0.01)$, peak flow $(+2.8 ; p<0.01)$ and VAS $(-6.5 ; p<0.01)$ with significant differences over placebo group (all p-value significant).

Conclusions: In this phase II clinical trial we showed the clinical efficacy of the treatment with Curcumin and Calendula in patients with CP/CPPS III. The benefits of this treatment could be related to the reduction of inflammatory cytokines and of inflammatory cells. These results should be confirmed in further studies with greater sample size.
\end{abstract}

KEY WORDS: Chronic prostatitis; Chronic pelvic pain syndrome type III; Pain; Prostate; Inflammation.

Submitted 21 November 2016; Accepted 11 January 2017

\section{INTRODUCTION}

Chronic prostatitis/chronic pelvic pain syndrome (CP/CPPS) is one of the most common disease of men under 50 and costly medical condition (1-4).

Unfortunately, the understanding of the underlying mechanisms is still lacking due the heterogeneous risks factors and associated conditions. Moreover, psychological condition and other trigger factors like infection or inflammation may act amplifying the CP/CPPS mechanisms. Based on this premise, CP/CPSS may negatively impact the quality of life (QoL) the psychological aspects of the patients. For all these reasons, the treatment strategies are different and may not necessarily determine a clinical efficacy, due the discrepancy in the treatment effects reported in case series and controlled trials that results from a large placebo effect or publication bias $(5,6)$. Among all therapies, phytotherapy has been largely used applying research to the practice of herbal medicine (7). The effect of such therapy is mainly based on the reduction of pain and in improving QoL, added to a mild anti-inflammatory effect. In this context, Calendula officinalis Linn. (Asteraceae) has been a subject of several chemical and pharmacological studies and it has been used especially for wound healing, jaundice and as an antispasmodic. Previously published results indicated that C. officinalis posses multiple pharmacological activities including antiinflammatory and antioedematous, antioxidant, immunostimulant, wound healing, hepatoprotective, antibacterial and antifungal and antiviral (8). In addition, numerous lines of evidence have indicated Curcuma longa (turmeric) ability in modulating multiple cell signaling molecules such as pro-inflammatory cytokines (tumor necrosis factor (TNF)- $\alpha$, interleukin (IL)-1 $\beta$, IL-6), apoptotic proteins, NF- $\kappa \mathrm{B}$, cyclooxygenase (COX)-2, STAT3, IKK $\beta$, endothelin-1, malondialdehyde (MDA). There are some evidences about rectal therapies with corticosteroids in patients with CP/CPSS, showing better drug's good safety profile and improving main storage symptoms and clinical findings associated with lower urinary tract inflammation in patients treated with beclomethasone di-propionate suppositories (9).

\section{MATERIALS AND METHOdS}

From June 2015 to January 2016 we enrolled 60 consecutive patients affected by CP/CPPS III in our institution. Patients between 20 and 50 year of age with symptoms of pelvic pain for 3 months or more before study according 
to European Association of Urology (EAU) guidelines, a negative 4-glass test Meares-Stamey test and a total National Institutes of Health Chronic Prostatitis Symptom Index (NIH-CPSI) score $\geq 15$ point were included in the study protocol. Patients diagnosed with NIH category IIIA and IIIB using the PPMT (pre- and post-massage test) have been enrolled. Category IIIA refers to the presence of white blood cells (WBC) after a prostate massage urine specimen (VB3) (WBC in VB3 > 10/hps). Category IIIB refers to patients with pelvic pain with no evidence of inflammation on VB3. Patients with urinary tract infection urethritis, sexually transmitted disease (STD), treatment with phytotherapeutic agents, alpha-blockers or antibiotics, urogenital cancer were excluded from the study.

At baseline subjects underwent uroflowmetry to evaluate the peak flow and the post void residual (PVR) and they filled out the International index of erectile function questionnaire (IIEF-5), the premature ejaculation diagnostic tool (PEDT) and the visual analogue score (VAS).

Patients were then randomized to receive treatment (Group A) or placebo (Group B). Treatment consisted of rectal suppositories of Curcumin extract $350 \mathrm{mg}$ (95\%) and Calendula extract $80 \mathrm{mg}$ (1 suppository/die for 1 month). Patients of Group B received 1 suppository/die for 1 month of placebo that exhibited identical characteristics of the treatment. Sample-size calculations were based on $80 \%$ power to detect a decline of 5 or more points in the NIH-CPSI total score between both groups, using a mean of the NIHCPSI of 25 and a standard deviation of 5 based on previous publication and requiring 24 subjects per group. The sample size has been set to 60 allowing a $15 \%$ of drop-out rate. The study has been approved the local ethic committee of the University of Catania. Clinical examination and questionnaires were collected after 3 months of therapy. All subjects gave written informed consent before entering the study, which was conducted in accordance with the Declaration of Helsinki, and the Human Ethics Committee approved the study protocol.

\section{Statistical analysis}

The primary endpoint of the study was the reduction of NIH-CPSI. The secondary outcomes were the change of peak flow, IIEF-5, VAS and of premature ejaculation diagnostic tool (PEDT). Sample-size calculations were based on $80 \%$ power to detect a decline of 5 or more points in the NIH-CPSI total score between both groups, using a mean of the NIH-CPSI of 25 and a standard deviation of 5 based on previous publication (Nickel et al.) (10) and requiring 24 subjects per group. The sample size has been set to 60 allowing a $15 \%$ of drop-out rate. The Mann-Whitney U Test was used for comparisons in the distribution of nonnormal variables between a pair of treatment. The efficacy variables were tested as change from baseline to 12 weeks relative to primary endpoints and secondary endpoints. A two-sided $p$-value $<0.05$ was considered statistically significant in all the tests used.

\section{RESULTS}

A total of 60 patients (median age of 32 years, interquartile range (IQR) 26-38) were enrolled in this study. The median of the duration of CP/CPPS was 9 months in both groups (IQR: 5-12). The flow chart of this study is presented in Figure 1. Of the 60 patients, 48 patients, 24 subjects in the group A and 24 patients in the group B respectively, completed the study protocol. Table 1 lists
Figure 1.

Flow chart of the study.

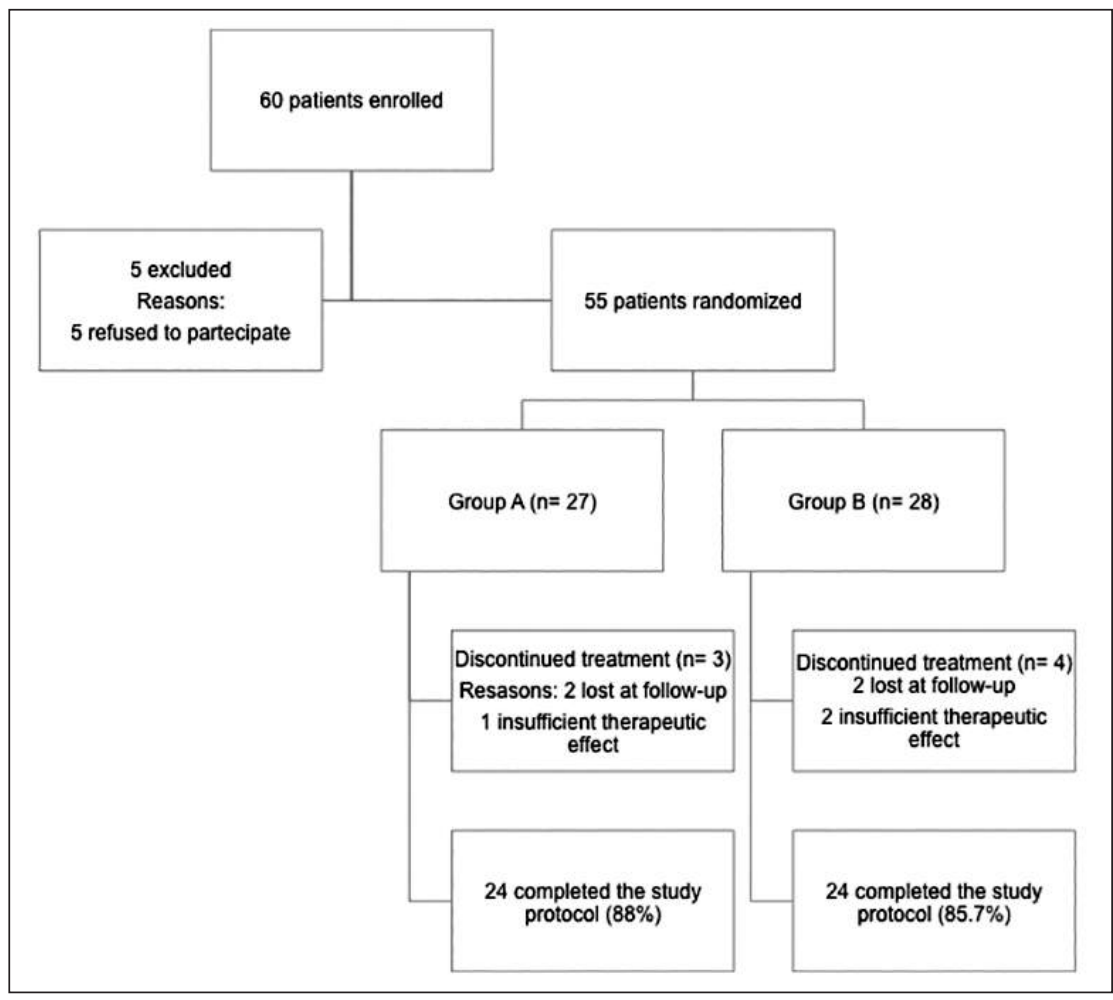

Table 1.

Characteristics of the enrolled patients at baseline.

\begin{tabular}{|lcc|}
\hline & $\mathbf{N}=\mathbf{2 4}$ & $\mathbf{N}=\mathbf{2 4}$ \\
\hline Age (years), median (IQR) & $32.0(29.00-38.0)$ & $32.0(28.75-38-75)$ \\
\hline NIH-CPSI, median (IQR) & $20.5(15.0-24.5)$ & $20.0(15.0-25.0)$ \\
\hline IIEF-5, median (IQR) & $18.5(17.50-21.00)$ & $18.5(17.5-21.5)$ \\
\hline PEDT, median (IQR) & $11.0(7.5-13.5)$ & $10.0(7.0-13.0)$ \\
\hline Peak flow (ml/s), median (IQR) & $14.0(10.5-19.5)$ & $14.5(10.5-19.5)$ \\
\hline PVR (ml), median (IQR) & $15.0(0.0-25.0)$ & $14.25(0.0-24.0)$ \\
\hline VAS, median (IQR) & $7.5(6.5-8.5)$ & $7.5(6.0-9.0)$ \\
\hline IQR = interquartile range; NIH-CPSI = NIH-Chronic Prostatitis Symptom \\
Index; IIEF-5 = International index of erectile function; \\
PEDT = premature ejaculation diagnostic tool; PVR = post void residual. \\
\hline
\end{tabular}


Figure 2.

Clinical characteristics of the patients after treatment.

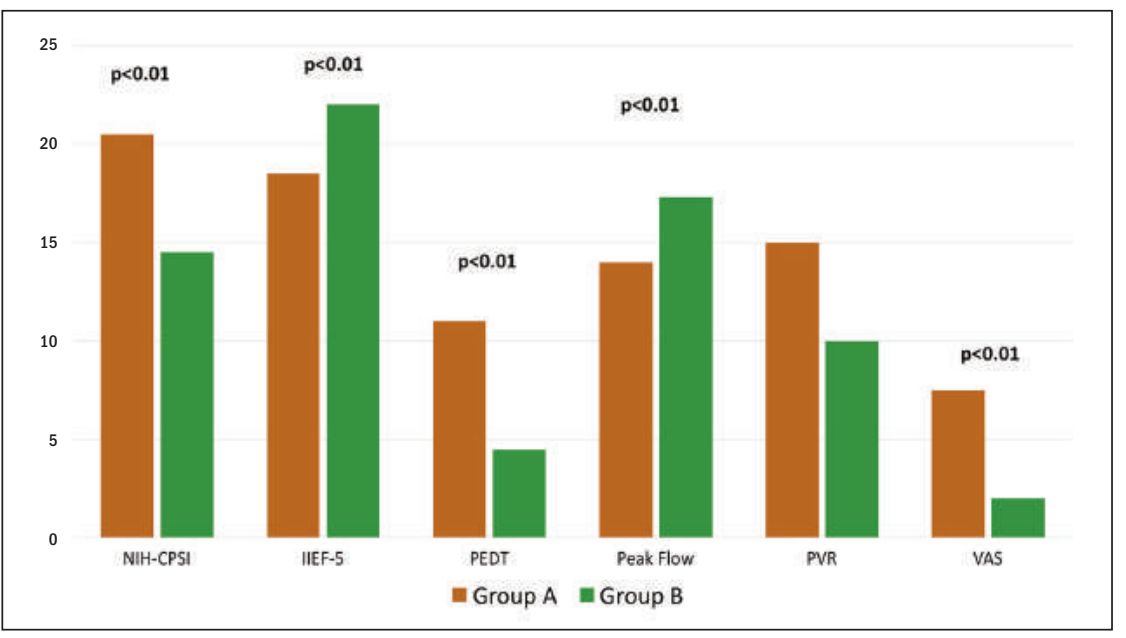

In fact, wide variety of therapies including alpha-blockers, antibiotics, anti-inflammatory medications, and other agents (eg, finasteride, phytotherapy, and gabapentinoids) are routinely used. However, the efficacy of these treatments is controversial, partly because many clinical trials testing these therapies have been small, with little statistical power to detect treatment effects (11).

Herein we showed that the treatment with rectal suppositories based on Curcumin extract $350 \mathrm{mg}$ and Calendula extract was clinical efficacious in terms of pain relief, voiding symptoms and urinary flow after 1 month of therapy and compared to placebo.

The rationale of such therapy could

the baseline characteristics of the patients at enrolment. No statistically significant differences emerged between groups at baseline in terms of collected variables.

In the group $\mathrm{B}$, the change from baseline to week 12 was significantly different relative to NIH-CPSI (median difference: $-5.5 ; \mathrm{p}<0.01$ ), IIEF-5 (median difference: +3.5 ; $\mathrm{p}<0.01$ ), PEDT (median difference: +5.5 ; $\mathrm{p}<0.01$ ), peak flow (median difference: +2.8 ; $\mathrm{p}<0.01$ ) and VAS score (mean difference: $-4.5 ; \mathrm{p}<0.01$ ). No significant difference was observed relative to PVR. In the group A we did not observed any changes from baseline to final follow-up relative to primary and secondary endpoints. When considering the comparison between both groups, we observed significant advantages of Group B over Group A regarding primary endpoint, IIEF-5, PEDT, peak flow and VAS (all $\mathrm{p}<0.01$ ) while any difference when considering PVR (Figure 2) (Table 2).

All subjects included in the study protocol tolerated treatments, but we observed a rate of adverse events of $5 \%$ in the Group B, including diarrhoea (80\%) and rectal pruritus (20\%)

\section{Discussion}

CP/CPPS represent one the most frequent disease in male under 50 and it is characterized by the heterogeneity of the risk factors and consequent clinical manifestation.

Table 2.

Characteristics of the enrolled patients after treatment.

\begin{tabular}{|lcc|}
\hline & $\mathbf{N}=\mathbf{2 4}$ & $\mathbf{N}=\mathbf{2 4}$ \\
\hline NIH-CPSI, median (SD) & $14.5(2.31)$ & $20.5(2.2)$ \\
\hline IIEF-5, median (SD) & $22.0(1.42)$ & $18.5(1.5)$ \\
\hline PEDT, median (SD) & $4.5(3.5)$ & $11(3.0)$ \\
\hline Peak flow (ml/s), median (SD) & $17.3(4.3)$ & $14(4.2)$ \\
\hline PVR (ml), median (SD) & $10.0(7.9)$ & $15(8.0)$ \\
\hline VAS, median (SD) & $2.0(1.9)$ & $7.5(2.0)$ \\
\hline IQR = interquartile range; NIH-CPSI = NIH-Chronic Prostatitis Symptom \\
$\begin{array}{l}\text { Index; IIEF-5 = International index of erectile function; } \\
\text { PEDT = premature ejaculation diagnostic tool; PVR = post void residual. }\end{array}$
\end{tabular}

be multiple and mainly related to their anti-inflammatory properties.

In this context,

Among the numerous natural remedies, turmeric has gained considerable attention due to its profound medicinal values (12).

This agent possesses antioxidant, anti-inflammatory, anticancer, antigrowth, anti-arthritic, anti-atherosclerotic, antidepressant, antiaging, antidiabetic, antimicrobial, wound healing and memory-enhancing activities (13). Curcumin acts against diverse range of molecular targets and signalling pathways. It can interact with a huge number of different proteins such as nuclear factor E2-related factor 2 (Nrf2), $\beta$-catenin, NF- $\kappa B$, p38 MAPK, DNA (cytosine-5)-methyltransferase-1, COX-2, 5-lipoxygenase, PGE2, FOXO3, inducible NOS, ROS, cyclin D1, VEGF, glutathione, cytosolic PLA2, p-Tau (p- $\tau$ ) and TNF- $\alpha$.

For all these reasons its use has gained popularity against several chronic conditions (14). In a recent meta-analysis of eight randomized clinical trials comprising nine treatment arms, curcumin was effective in reducing circulating TNF- $\alpha$ concentrations (WMD: -4.69 p < 0.001) (15). Curcumin has been also combined with other compounds like quercetin and prulifloxacin for the treatment of chronic bacterial prostatitis, showing improvement of clinical efficacy of prulifloxacin (16).

On the other part, Calendula officinalis flower extract possessed significant anti-inflammatory activity. In chronic anti-inflammatory model using formalin, administration of 250 and $500 \mathrm{mg} / \mathrm{kg}$ body weight Calendula extract produced an inhibition of 32.9 and $62.3 \%$ respectively compared to controls. TNF-alpha production by macrophage culture treated with lipopolysaccharide (LPS) was found to be significantly inhibited by Calendula extract.

Moreover, increased levels of pro-inflammatory cytokines IL- Ibeta, IL-6, TNF-alpha and IFN-gamma and acute phase protein, $\mathrm{C}$ - reactive protein (CRP) in mice produced by LPS injection were inhibited significantly by the extract. LPS induced cyclooxygenase- 2 (Cox-2) levels in mice spleen were also found to be inhibited by extract treatment (17).

However, there are not any comparison between these 
compounds and other for the treatment of CP/CPSS. In a meta-analysis published on 2011 on three studies comparing phytotherapy with placebo $(n=223)$, authors demonstrated a reduction of pain score (standard mean difference (SMD): -0.5) and in voiding symptoms (SMD: -0.4). Moreover, phytotherapy ehibited the second highest response rate after alpha-blockers, showing a risk ratio of 1.6 compared with placebo (11).

Before concluding some limitations should be addressed. First of all, it is a pilot, phase II study with a small sample size. Moreover, we did not investigate marker of inflammation in the cohort.

On the contrary, some strengths of the study are the investigation of new compounds for the treatment of CP/CPPS and the randomized placebo controlled design. Further study with greater sample size should conducted to confirm these data.

\section{Conclusions}

In this phase II, placebo controlled, clinical trial we demonstrated the efficacy of rectal suppositories with Curcumin and Calendula for the treatment of CP/CPPS, in terms of pain and urinary symptoms.

\section{REFERENCES}

1. Rees J, Abrahams M, Doble A, Cooper A, Prostatitis Expert Reference G. Diagnosis and treatment of chronic bacterial prostatitis and chronic prostatitis/chronic pelvic pain syndrome: a consensus guideline. BJU Int. 2015; 116:509-25.

2. Morgia G, Cimino S, Favilla V, et al. Effects of Serenoa repens, selenium and lycopene (Profluss $(R))$ on chronic inflammation associated with benign prostatic hyperplasia: results of "FLOG" (Flogosis and Profluss in Prostatic and Genital Disease), a multicentre Italian study. Int Braz J Urol. 2013; 39:214-21.

3. Morgia G, Mucciardi G, Gali A, et al. Treatment of chronic prostatitis/chronic pelvic pain syndrome category IIIA with Serenoa repens plus selenium and lycopene (Profluss) versus S. repens alone: an Italian randomized multicenter-controlled study. Urol Int. 2010; 84:400-6.

4. Russo GI, Cimino S, Fragala E, et al. Relationship between nonalcoholic fatty liver disease and benign prostatic hyperplasia/lower urinary tract symptoms: new insights from an Italian cross-sectional study. World J Urol. 2015; 33:743-51.

5. Engeler DS, Baranowski AP, Dinis-Oliveira P, et al. The 2013 EAU guidelines on chronic pelvic pain: is management of chronic pelvic pain a habit, a philosophy, or a science? 10 years of development. Eur Urol. 2013; 64:431-9.

6. Vanella L, Russo GI, Cimino S, et al. Correlation between lipid profile and heme oxygenase system in patients with benign prostatic hyperplasia. Urology. 2014; 83:1444 e7-13.

7. Morgia G, Russo GI, Voce S, et al. Serenoa repens, lycopene and selenium versus tamsulosin for the treatment of LUTS/BPH. An Italian multicenter double-blinded randomized study between single or combination therapy (PROCOMB trial). Prostate. 2014; 74:1471-80.

8. Zaki A, Ashour A, Mira A, et al. Biological Activities of Oleanolic Acid Derivatives from Calendula officinalis Seeds. Phytother Res. 2016; 30:835-41.

9. Bozzini G, Provenzano M, Buffi N, et al. An observational study of the use of beclomethasone dipropionate suppositories in the treatment of lower urinary tract inflammation in men. BMC Urol. 2016; 16:25.

10. Nickel JC, Krieger JN, McNaughton-Collins M, et al. Alfuzosin and symptoms of chronic prostatitis-chronic pelvic pain syndrome. N Engl J Med. 2008; 359:2663-73.

11. Anothaisintawee T, Attia J, Nickel JC, et al. Management of chronic prostatitis/chronic pelvic pain syndrome: a systematic review and network meta-analysis. JAMA. 2011; 305:78-86.

12. Prasad S, Gupta SC, Tyagi AK, Aggarwal BB. Curcumin, a component of golden spice: from bedside to bench and back. Biotechnol Adv. 2014; 32:1053-64.

13. Gupta SC, Patchva S, Aggarwal BB. Therapeutic roles of curcumin: lessons learned from clinical trials. AAPS J. 2013; 15:195-218

14. Kunnumakkara AB, Bordoloi D, Padmavathi G, et al. Curcumin, the golden nutraceutical: multitargeting for multiple chronic diseases. Br J Pharmacol. 2016 Sep 17. doi: 10.1111/bph.13621. (Epub ahead of print)

15. Sahebkar A, Cicero AF, Simental-Mendia LE, et al. Curcumin downregulates human tumor necrosis factor-alpha levels: A systematic review and meta-analysis ofrandomized controlled trials. Pharmacol Res. 2016; 107:234-42.

16. Cai T, Mazzoli S, Bechi A, et al. Serenoa repens associated with Urtica dioica (ProstaMEV) and curcumin and quercitin (FlogMEV) extracts are able to improve the efficacy of prulifloxacin in bacterial prostatitis patients: results from a prospective randomised study. Int J Antimicrob Agents. 2009; 33:549-53.

17. Preethi KC, Kuttan G, Kuttan R. Anti-inflammatory activity of flower extract of Calendula officinalis Linn. and its possible mechanism of action. Indian J Exp Biol. 2009; 47:113-20.

\author{
Correspondence \\ Giuseppe Morgia, MD \\ Giorgio Ivan Russo, MD, PhD (Corresponding Author) \\ giorgioivan@virgilio.it \\ Daniele Urzi, MD \\ Salvatore Privitera, MD \\ Tommaso Castelli, MD \\ Vincenzo Favilla, MD \\ Sebastiano Cimino, MD \\ Urology section, Department of Surgery, University of Catania, Italy
}

